\title{
Reverse trigger finger: does it exist? a case report
}

\begin{abstract}
Trigger finger is a common condition, occurs primarily at the A1 pulley and tenosynovitis is the usual cause. However, although rare, triggering of the flexor tendon can occur in other areas. We present previously unreported condition in adult: triggering at the distal end of A2 pulley, which was caused by nodular thickening of the flexor digitorium profondus tendon with degeneration. A 79-year-old lady presented with atypical presentation of trigger finger at the distal end of A2 pulley. Intra-operatively a nodular thickening of the flexor digitorium profondus tendon with degeneration was found, which was similar to a notta nodule. A sample of the tendon was sent for histology, which showed a degenerative cystic structure. The A2 pulley was therefore fully released and reduction tenoplasty was performed distal to A2 pulley. We hope that by presenting this case we will increase awareness of such presentation of trigger finger leading to improved diagnosis and treatment of the process.
\end{abstract}

Volume 6 Issue 6 - 2016

\author{
Sabri Bleibleh,' Mariana Abdel-Malek, ${ }^{2}$ \\ Richard Dias, ${ }^{2}$ Sukhraj Dhillon ${ }^{3}$ \\ 'Medway Maritime Hospital, United Kingdom \\ ${ }^{2} \mathrm{New}$ Cross Hospital, United Kingdom
}

\begin{abstract}
Correspondence: Sabri Bleibleh MBBS, MRCSEd, ST3 Trauma and Orthopaedics registrar, Medway Maritime Hospital, United Kingdom, ME7 5NY,Tel 448000000000,

Email shabri_bleibleh@yhoo.com
\end{abstract}

Received: August 29, 2016 | Published: December 09, 2016

Keywords: Hand, Trigger finger, Trigger Digit, Reverse, A2 Pulley

\section{Introduction}

Notta initially described trigger finger or stenosing tenosynovitis of the digital flexor sheath in 1850. It is one of the most common pathologies presented at hand clinics, with a lifetime risk of $2.6 \%$ within the general population. ${ }^{1,2}$ The incidence varies according to the patient population; it is significantly higher amongst diabetics and females, yet prevalent in only $0.3 \%$ of children. ${ }^{1}$ The condition is characterised by the restricted motion of the digit, painful clicking and subsequent locking of the digit. This usually occurs a result of a failure in the first annular (A1) pulley gliding mechanism, due to a discrepancy in the diameter between the flexor tendon and its inflamed, hypertrophic sheath. ${ }^{2}$ It is a typically straightforward diagnosis that is initially managed with steroid injections. ${ }^{2,3}$

We, however, report an unusual presentation of trigger finger with an extremely rare site of pathology at the level of the second annular (A2) pulley; a case previously unreported in adults.

\section{Case report}

A 79 year-old, right-handed female presented with a three to four year history of swelling and triggering over the Proximal InterPhalangeal Joint (PIPJ) of which had become worse over the last two years, with restriction of movement of the right index finger and inability to fully flex the PIPJ and Distal Inter-Phalangeal Joint (DIPJ). This condition affected her daily activities, which she found very frustrating. She had no beneficial response to a steroid injection. There was no previous history of trauma.

On physical examination, a palpable, painless, tense swelling was felt over the palmar aspect of the index finger PIPJ. Triggering was confirmed at the level of A2 pulley on flexion. No evidence of neurological or vascular deficit.

Surgical exploration was carried out under a regional block. The flexor tendon sheath was exposed by a bruner's incision, which was made over the volar aspect of the index finger PIPJ. Intra-operatively a nodular thickening of the Flexor Digitorium Profondus (FDP) tendon with degeneration was found, which was similar to a notta nodule (Figure 1). The nodule was getting trapped in the distal mouth of the A2 pulley. There was no or a very thin looking A3 pulley which was very degenerate (Figure 2). The A2 pulley was therefore fully released and reduction tenoplasty was performed distal to A2 pulley (Figure 3). A sample was excised from the flexor tendon and sent for histology, which showed a cystic structure. The flexor tendon was examined and revealed no other pathology. After the release of the A2 pulley, the patient was able to fully flex the DIP and PIP joints and make a full fist. She made an uneventful recovery.

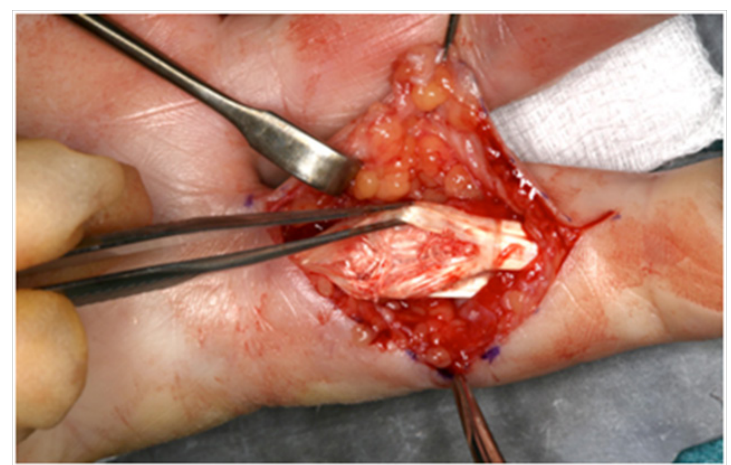

Figure I Nodular thickening of the FDP tendon with degeneration, similar to a notta nodule.

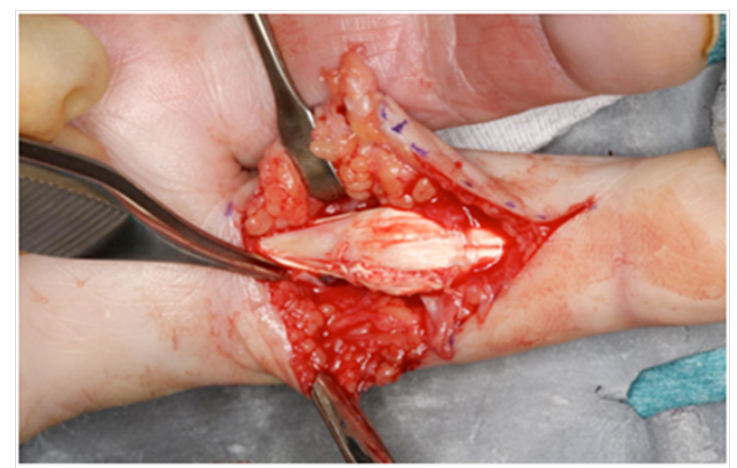

Figure 2 Thin and degenerative A3 pulley.

\section{Discussion}

Mechanical strain secondary to repetitive finger movement is a well-recognised cause of triggering the digital flexor tendon. ${ }^{4}$ The annular pulleys are located at the foci of highest force from the flexor tendons. ${ }^{4}$ Hence, the increased occurrence of stenosing tenosynovitis at the A1 pulley region is unsurprising in view of its anatomical location at the metacarpal head. This positioning subjects it to maximal stress from the friction generated by repetitive flexion and extension within both a relaxed, and power handgrip. ${ }^{2,3}$ The 
inflammatory process and swelling that ensues thereby leads to loss of motion in the offending digit. Triggering of the A2 pulley system is significantly less common. Nonetheless, the distal portion of the A2 pulley is thicker than its proximal counterpart, which is a potential, albeit rare, anatomical source of entrapment. .,6,7 $^{5}$

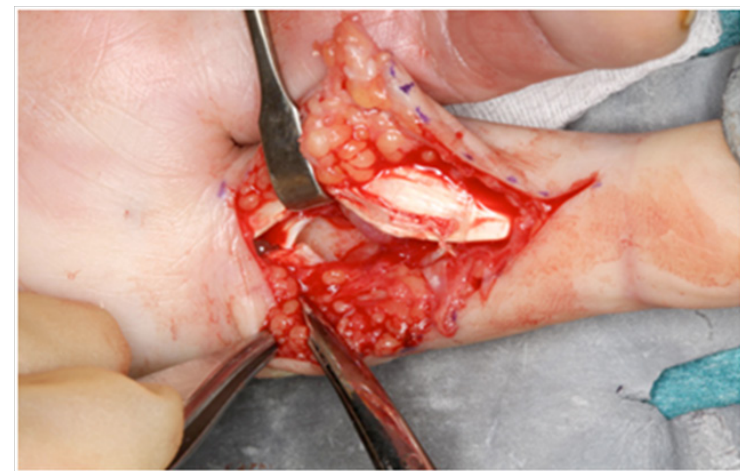

Figure $3 \mathrm{~A} 2$ pulley was fully released and tenoplasty of the FDP tendon distal to $\mathrm{A} 2$.

In this case, although the patient was restricted in flexion at the DIPJ of the affected finger, there was no clinical evidence of triggering at either the first, or even second, annular pulley. Hence, the intra-operative finding was highly incidental to the clinical picture. Moreover, as the site of pathology at the A2 pulley is extremely rare, it has only been cited occasionally throughout the literature and largely as an early-age feature. Hirata et al. ${ }^{1}$ reported the unique finding of a Notta's nodule at the A2 pulley in one such case of congenital triggering. Ikeda \& Osamura. ${ }^{5}$ identified two further cases of triggering at the distal and the proximal entry of the A2 pulley, causing a loss of flexion or extension in the affected digit, respectively. Although these reports were also in children, similarities arose from the finding of a nodule of FDP tendon and the success of surgical treatment.

Throughout the few cases reported, the flexor tendon involved in triggering at the A2 pulley has varied, although the flexor digitorum superficialis tendon is most often implicated. ${ }^{8}$ In our case, the triggering occurred subsequent to the nodular thickening and enlargement of the FDP tendon as it was pulled into the distal mouth of the A2 pulley. Following the release of the patient's A2 pulley, she was able to fully flex the DIPJ and PIPJ, which essentially enabled her to make a full fist. This clinical effect further corroborates the rare mechanism portrayed that underlies a common pathology. Nagaoka et al. ${ }^{6}$ have similarly reported a case of entrapment at the A2 pulley from an enlarged FDP tendon. However, unlike with our patient, the triggering was not fully relieved by a reduction tenoplasty and a distal venting of the A2 pulley was required.
In such an atypical presentation, further imaging of the finger in question would have been warranted. Magnetic resonance or ultrasonographic imaging could have initially excluded other differentials of a locked finger including Duputyren's contracture, post-traumatic entrapment or a tumour of the flexor tendon. ${ }^{2}$ An oncogenic basis of trigger finger, although extremely rare, has been identified amongst reports within the literature; including a leiomyoma, chondroma, neurilemmoma and giant cell tumour., Although there was no evidence of such pathology on surgical evaluation, a sample was sent nonetheless for histological analysis. We hope that by presenting this case we will increase awareness of such presentation of trigger finger leading to improved diagnosis and treatment of the process. ${ }^{10}$

\section{Acknowledgments}

None.

\section{Conflicts of interest}

None.

\section{References}

1. Hirata H, Fujisawa K, Sasaki $\mathrm{H}$ et al. Congenital triggering of the index finger at the A2 pulley. J Hand Surg. 1996;21(5):609-611.

2. Makkouk AH, Oetgen ME, Swigart $\mathrm{CR}$ et al. Trigger finger: etiology, evaluation, and treatment. Curr Rev Musculoskelet Med. 2008;1(2):92-96.

3. Akhtar S, Bradley MJ, Quinton DN Management and referral for trigger finger/thumb. BMJ 2005;331(7507):30-33.

4. Moore JS Flexor tendon entrapment of the digits (trigger finger and trigger thumb). J Occup Environ Med. 2000;42(5):526-545.

5. Ikeda $\mathrm{K}$, Osamura $\mathrm{N}$ Trigger finger at the $\mathrm{A} 2$ pulley in children -2 case reports. J Plast Reconstr Anesthet Surg. 2010;63(8):e635-6.

6. Nagaoka M, Yamaguchi T, Nagao S Triggering at the distal A2 pulley. $J$ Hand Surg Eur 2007;32(2):210-213.

7. Walbeehm ET, McGrouther DA An anatomical study of the mechanical interactions of flexor digitorum superficialis and profundus and the flexor tendon sheath in zone 2. J Hand Surg Br. 1995;20(3):269-280.

8. Wood VE, Sicilia M Congenital trigger digit. Clin Orthop Relat Res. $1992 ;(285): 205-209$.

9. Harb Z, Bismil Q, Ricketts DM Trigger finger presenting secondary to leiomyoma: a case report. J Med Case Rep. 2009;8(3):7284.

10. Choudhury MM, Tay SC Prospective study on the management of trigger finger. Hand Surg. 2014;19(3):393-397. 